\title{
Original
}

\section{Sleep Apnea in Rheumatoid Arthritis Patients with Atlantoaxial Subluxation}

\author{
Kazunobu IshIKAWA ${ }^{1,2)}$, Katsunori INAGAKI ${ }^{1)}$, Osamu NAMIKI ${ }^{1}$, \\ Etsuo FuJIMAKI ${ }^{1)}$ and Ikuo Homma ${ }^{2)}$
}

\begin{abstract}
Obstructive sleep apnea has been reported in rheumatoid arthritis (RA) patients who have lesions of the upper cervical vertebrae. We studied the pathophysiology of sleep apnea in RA patients with atlantoaxial subluxation by monitoring movements of the chest wall and abdomen, airflow, and arterial blood oxygen saturation during sleep. Nine female RA patients aged 49 to 80 years took part in this experiment. Five of them were in stage III, and four of them were in stage IV. Anterior subluxation was seen in six patients, and posterior subluxation was seen in three patients. Sleep apnea syndrome, diagnosed when the apnea index was more than 5, was observed in seven of the nine patients. The three patients with posterior atlantoaxial subluxation had high apnea indices $(87.7,48.6$, and 12.6). Central sleep apnea with absence of airflow and chest wall and abdominal wall movements was observed in two patients with posterior atlantoaxial subluxation. In these patients the anterior arc of the atlas was dislocated posteriorly. Compression of the ventral part of the lower brainstem and the upper cervical spinal cord was also observed. These results suggest that central apnea might be induced when the structures in the caudal part of the ventral medulla are blocked. The dysfunction of respiratory structures might occur in RA patients with posterior atlantoaxial subluxation.
\end{abstract}

Key words: central apnea, obstructive sleep apnea, ventral medulla

\section{Introduction}

Rheumatoid arthritis (RA) is regarded as an autoimmune disease. After Sharp et al. (1958) demonstrated the destruction of cervical vertebra in RA, the possibility of upper cervical spinal cord injuries has been a cause of concern ${ }^{1)}$. Recent studies have shown that patients who have upper cervical spinal cord injuries show symptoms of sleep apnea ${ }^{2,3}$. Burwell et al. (1956) reported that sleep apnea occurs in obese people who tend to stop breathing while sleeping as a result of airway obstruction ${ }^{4}$. This condition was named sleep apnea syndrome (SAS) ${ }^{5)}$. This is also called Pickwickian syndrome, after Dicken's novel. According to animal experiments, apnea has two causes. One cause is airway obstruction, and the other cause is a central abnormality ${ }^{6}$. Although SAS has been suggested to be induced by light spinal cord compression in patients with atlantoaxial subluxation, it is not known whether SAS in RA patients with atlantoaxial subluxation is caused by a cen-

\footnotetext{
1) Department of Orthopedic Surgery, Showa University School of Medicine, 1-5-8 Hatanodai, Shinagawaku, Tokyo 142, Japan.

2) Second Department of Physiology, Showa University School of Medicine.
} 
ADI

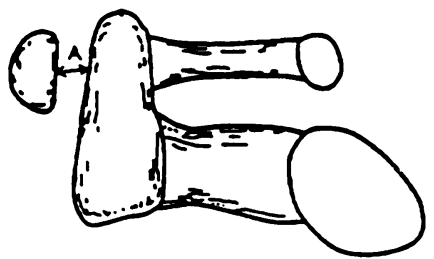

Ranawat score

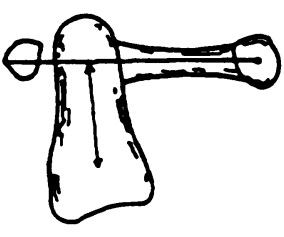

Redlund-Johnell score

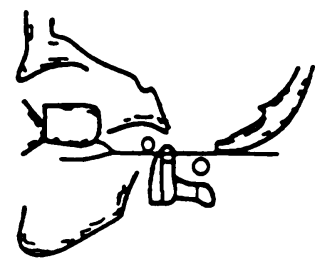

Fig. 1. Calculations of atlantodental interval (ADI), Ranawat score and Redlund-Johnell score.

tral abnormality or by obstruction.

The purpose of the present study was to investigate the relationship between SAS and compression of the upper cervical spinal cord and lower brainstem in RA patients by monitoring and analyzing respiration during sleep.

\section{Methods}

Subjects: Nine female RA patients aged from 49 to 80 years with upper cervical subluxation took part in this experiment. Their breathing was monitored during sleep. The pathologic stage of RA was stage III in 5 patients and stage IV in 4 patients, of whom 1 was in class 2, 4 were in class 3 , and 4 were in class 4 .

Procedure and design: Airflow was monitored by a thermistor attached to the subject's nose. Two strain gauges were attached to the chest and the abdominal wall to measure chest wall movement and abdominal movement. Arterial oxygen saturation $\left(\mathrm{SaO}_{2}\right)$ was measured by a pulse oximeter (N 20P, Nihon Koden, Tokyo, Japan) attached to the index finger. Both electroencephalograms and electrooculograms were recorded by a pair of surface electrodes attached to the scalp and the skin over the orbicularis oculi muscle. All data during the night were recorded with an eight-channel rectilinear polygraph (RTA-1200, Nihon Koden). The apnea index was the number of times breathing stoped for more than 10 seconds in 1 hour; when the apnea index was more than 5, SAS was diagnosed.

Obstructive sleep apnea was diagnosed when airflow was absent and chest wall and abdominal movements continued. Central apnea was diagnosed when both airflow and chest and abdominal movements were absent.

The upper cervical spinal cord and the lower brainstem were examined with X-ray and magnetic resonance imaging. From the X-ray images the atlantodental interval (ADI), Ranawat score, and Redlund-Johnell (R-J) score were calculated (Fig. 1).

\section{Results}

Table 1 presents the ADI ( $\mathrm{mm})$, Ranawat scores $(\mathrm{mm})$, Redlund-Johnell scores $(\mathrm{mm})$, and the apnea index with patients' age, disease stage, and disease class. Horizontal atlantoaxial subluxation was seen in each of the nine RA patients. Anterior atlantoaxial subluxation was seen in six RA patients with an ADI of more than $3 \mathrm{~mm}$, and posterior subluxation was seen in three RA patients. A Ranawat score of less than $13 \mathrm{~mm}$, which indicates vertical subluxation, was seen in five patients. A R-J score also indicating vertical subluxation was 
Table 1. Patient profiles

\begin{tabular}{cccccccc}
\hline Case & Age & Stage & Class & ADI $(\mathrm{mm})$ & $\begin{array}{c}\text { Ranawat } \\
(\mathrm{mm})\end{array}$ & $\begin{array}{c}\text { R-J } \\
(\mathrm{mm})\end{array}$ & Apnea index \\
\hline 1 & 61 & III & 4 & Posterior subluxation & 10 & 23 & 87.7 \\
2 & 49 & III & 3 & Posterior subluxation & 14 & 27 & 48.6 \\
3 & 49 & III & 3 & 3 & 3 & 25 & 17.6 \\
4 & 80 & IV & 4 & Posterior subluxation & 3 & 16 & 12.6 \\
5 & 67 & IV & 4 & 4 & 0 & -3 & 9.0 \\
6 & 67 & IV & 3 & 3 & 15 & 25 & 8.0 \\
7 & 61 & IV & 4 & 11 & 14 & 46 & 5.0 \\
8 & 60 & III & 2 & 5 & 16 & 27 & 2.0 \\
9 & 61 & III & 3 & 6 & 8 & 25 & 0 \\
\hline
\end{tabular}

ADI, atlantodental interval; R-J, Redlund-Johnell score.

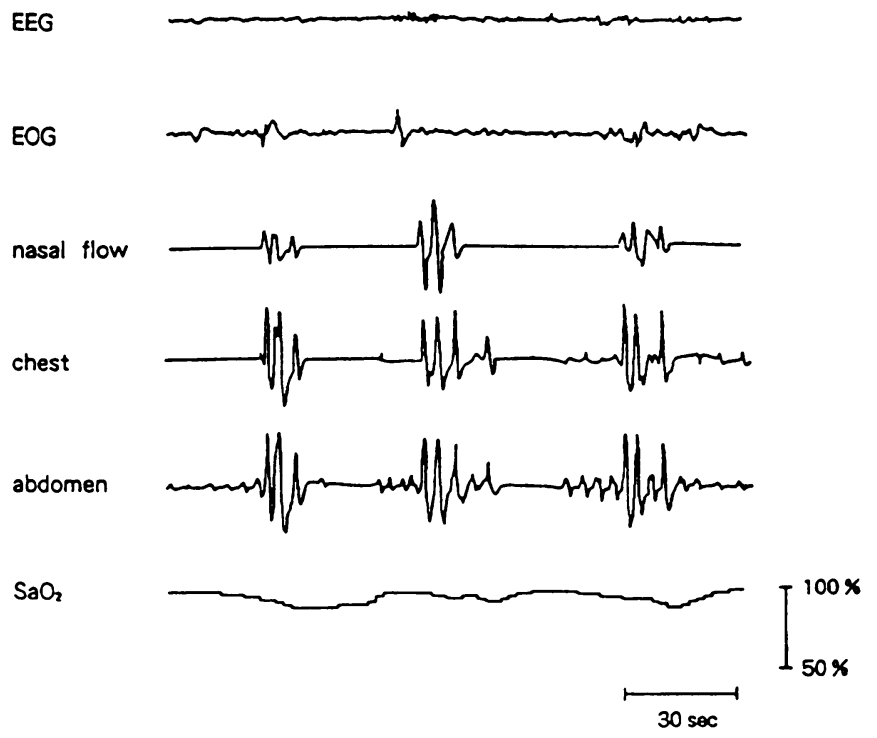

Fig. 2. Polygraph recordings in a patient (case 1) during sleep. EEG, electroencephalography; EOG, electrooculography; chest, chest wall movement; abdomen, abdominal wall movement. Upper deflection indicates inspiration.

seen in eight patients. SAS with an apnea index of more than 5 was observed in seven of nine patients. Among the 7 patients with SAS, the apnea index was from 5 to 9 in 3 patients, from 10 to 20 in 2 patients, and more than 30 in 2 patients (Table 1). Comparison of lesions on X-ray and apnea indices indicated that the three patients with posterior atlantoaxial subluxation tended to have higher apnea indices $(87.7,48.6$, and 12.6). There was no correlation between stage or class and the apnea index.

Central sleep apnea with absence of airflow and absence of movements of the chest wall and abdominal wall was observed in two of seven patients who had SAS. Both patients had posterior atlantoaxial subluxation (Fig. 2). Ventilation alternated repeatedly with apnea. During periods of apnea, airflow was only absent when the chest wall and abdominal wall 


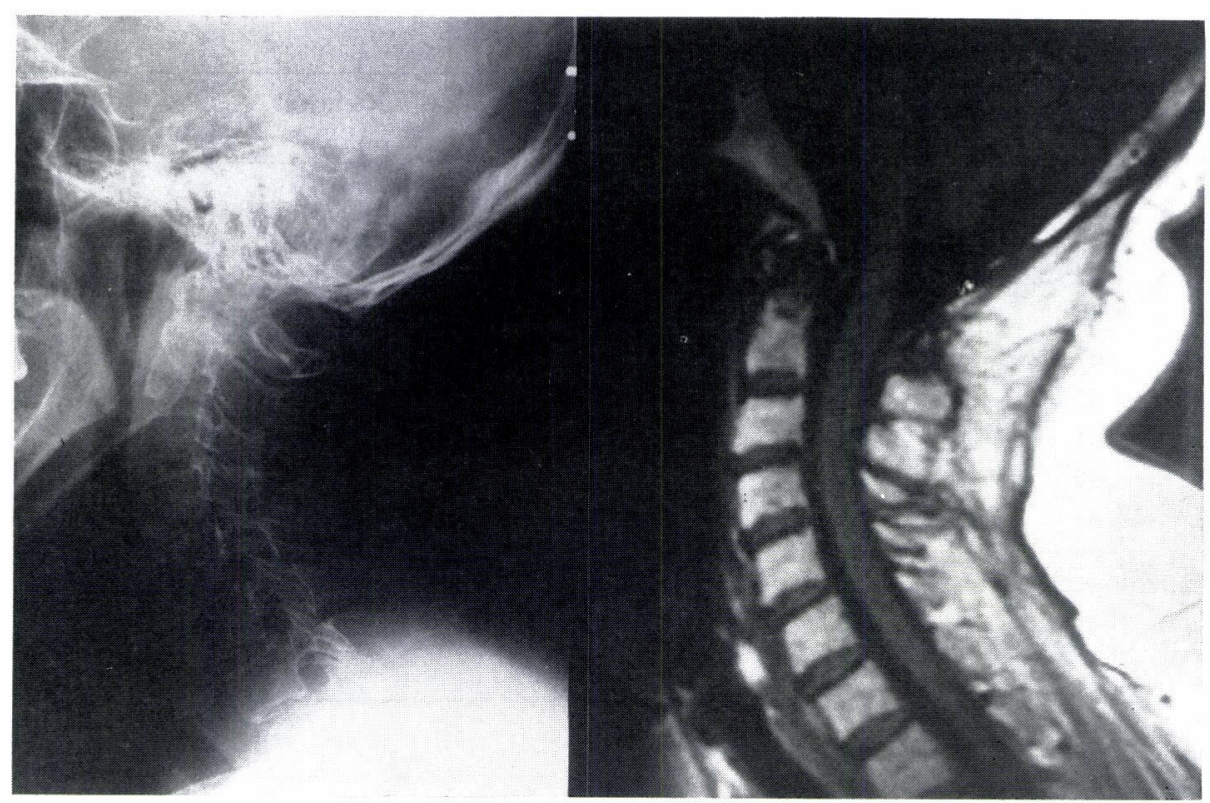

Fig. 3. X-ray (left) and MRI (right) images in a patient (case 1). Posterior subluxation and compression of the caudal medulla was seen.

were continuously moving. Episodes of apnea lasted more than 1 minute but varied in duration. The $\mathrm{SaO}_{2}$ was as low as $53 \%$.

In X-rays showing posterior atlantoaxial subluxation, the anterior arc of the atlas was dislocated posteriorly. The synovial membrane around the dens was also increased. The ventral part of the lower brainstem and the upper cervical spinal cord were also compressed in these patients (Fig. 3).

\section{Discussion}

The atlas is held only by ligaments so that it is more mobile than other cervical vertebrae. Six of nine RA patients in this study had anterior atlantoaxial subluxation, and the other three patients had posterior subluxation.

Davies and Iber first reported obstructive sleep apnea in RA patients in 19837). However, this airway obstruction was induced by micrognathia due to destruction of the temporomandibular joint. The upper airway obstruction caused by destruction of the temporomandibular joint has been reported to induce obstructive sleep apnea ${ }^{8)}$. One cause of this obstructive sleep apnea is compression of the airway in obese patients with excessive cervical adipose tissue. Another cause is airway obstruction due to tonsillar enlargement or endocrine disturbances ${ }^{9,10)}$. Airway obstruction can also result from dysfunction of the genioglossus muscle $^{11)}$. When this muscle is weak, the airway is compressed by contraction of the muscles of inspiration. In patients with micrognathia, the contractile force of the muscles of inspiration surpasses that of the genioglossous muscle ${ }^{7}$.

In this study we showed two types of sleep apnea in RA patients: obstructive apnea and central apnea. However, central sleep apnea was observed in patients with posterior atlantoaxial subluxation. It has been reported that only $2.8 \%$ of atlantoaxial subluxation 


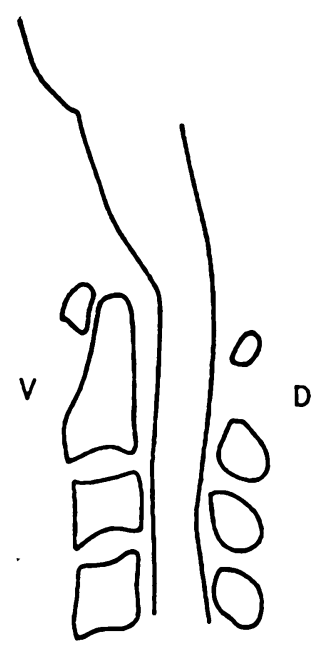

N

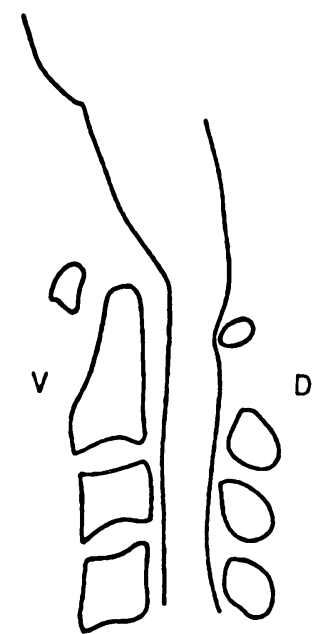

AS

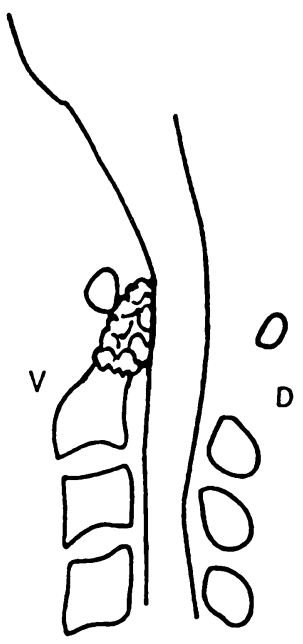

PS

Fig. 4. Schematic representation of compression of the ventral part of caudal medulla and upper cervical spinal cord. V, ventral; D, dorsal; N, normal; AS, anterior subluxation; PS, posterior subluxation. Ventral compression is obvious in PS.

in RA is posterior atlantoaxial subluxation ${ }^{12)}$. Most causes of posterior atlantoaxial subluxation are caused by the fossa dentis forcibly compressing the anterior joint of the dentis. Erosion of the dens commonly occurs in mutilans types of RA. In animal experiments, Homma et al. showed that focal cold block of the ventral structures in the medulla oblongata stops respiration $^{6)}$. Focal cold block in the caudal part of the ventral medulla 0 to $1 \mathrm{~mm}$ rostral from the obex and $4 \mathrm{~mm}$ beneath the dorsal surface, strongly reduced respiratory frequency with little change in the shape or size of inspiratory activities in rabbit. Rostral focal cold block suppressed tidal inspiratory activity with increased respiratory frequency. Homma et al. called the former type of central apnea 'temporal apnea' and the latter type 'suppression apnea's). They also recorded respiratory pacemaker-type neural activity in the ventrolateral medulla in a neonatal rat brainstem-spinal cord preparation ${ }^{13)}$. Furthermore, they emphasized the importance of the ventrolateral medulla as an respiratory rhythm generator.

The ventral parts of upper cervical spinal cord and the lower medulla were compressed by posterior atlantoaxial subluxation (Figs. 3 and 4 ). The central sleep apnea observed in these patients was considered 'temporal apnea' as shown by Homma et al. in animal experiments. This type of apnea is induced when structures in the caudal part of the ventral medulla are blocked.

We have not proven that dysfunction of respiratory structures in the caudal ventral medulla exists in these RA patients with SAS. However, the results obtained in this study suggest the possibility of respiratory dysfunction in the medulla.

\section{References}

1) Sharp J, Purser DW and Lawrence JS: Rheumatoid arthritis of the cervical spine in the adult. Ann Rheum Dis, 17: 303-313 (1958)

2) Hall $\mathrm{CW}$ and Danoff $\mathrm{D}$ : Sleep attacks: apparent relationship to atlantoaxial dislocation. Arch Neurol, 32: 57-58 (1975) 
3) Balk RA, Hiller FC, Lucas EA, Scrima L, Wilson FJ and Wooten V: Sleep apnea and the ArnoldChiari malformation. Am Rev Respir Dis, 132: 929-930 (1985)

4) Burwell CS, Robin ED, Whaley RD and Bickelmann AG: Extreme obesity associated with hypoventilation-A Pickwickian syndrome. Am J Med, 21: 811-824 (1956)

5) Guilleminault C, Van der HJ and Mitler MM: Clinical overviews of the sleep apnea syndromes. In: Sleep Apnea Syndrome, Guilleminault C and Dement W (Eds). Alan R Liss, Inc., New York, pp 1-12 (1978)

6) Homma I, Isobe A, Iwase M, Kanamaru A and Sibuya M: Two different types of apnea induced by focal cold block of ventral medulla in rabbits. Neurosci Lett, 87: 41-45 (1988)

7) Davies SF and Iber $C$ : Obstructive sleep apnea associated with adult-acquired micrognathia from rheumatoid arthritis. Am Rev Respir Dis, 127: 245-247 (1983)

8) Redlund-Johnell I: Upper airway obstruction in patients with rheumatoid arthritis and temporomandibular joint destruction. Scand J Rheumatol, 17: 273-279 (1988)

9) Orr WC and Martin RJ: Obstructive sleep apnea associated with tonsillar hypertrophy in adults. Arch Intern Med, 141: 990-992 (1981)

10) Zwillich CW, Pierson DJ, Hofeldt FD, Lufkin EG and Weil JY: Ventilatory control in myxedema and hypothyroidism. $N$ Engl J Med, 292: 662-665 (1975)

11) Remmers JE, deGroot WJ, Sauerland EK and Anch AM: Pathogenesis of upper airway occlusion during sleep. J Appl Physiol, 44: 931-938 (1978)

12) Redlund-Johnell I: Posterior atlanto-axial dislocation in rheumatoid arthritis. Scand J Rheumatol, 13: 337-341 (1984)

13) Onimaru H, Arata A and Homma I: Primary respiratory rhythm generator in the medulla of brainstem-spinal cord preparation from newborn rat. Brain Res, 445: 314-324 (1988)

[Received February 16, 1996: Accepted February 22, 1996] 